\title{
ON RADIO SQUARE SUM D-DISTANCE NUMBER OF GRAPHS
}

\author{
K. JOHN BOSCO ${ }^{1}$ \& G.VISHMA GEORGE ${ }^{2}$ \\ ${ }^{I}$ Assistant professor, Department of Mathematics, St. Jude's College, Thoothoor, Tamil Nadu, India \\ ${ }^{2}$ Research scholar, Manonmaiumsundaranar University, St. Jude's College, Thoothoor, Tamil Nadu, India
}

\begin{abstract}
Let $d^{D}(u, v)$ denotes the D-distance between two distinct vertices of a connected graph $G$, and $\operatorname{diam}^{D}(G)$ be the D-diameter of $G$. A Radio square sum D-distance coloring of a connected graph $G$ is an one-to-one mapping $\quad c: V(G) \rightarrow N$ such that condition holds every two distinct vertices $u$ and $v d^{D}(u, v)+$ $\left|[c(u)]^{2}+[c(v)]^{2}\right| \geq 1+\operatorname{diam}^{D}(G) .$. The $\operatorname{rssn}^{D}(c)$ is the maximal span of $G$ and $\operatorname{rssn}^{D}(G)$ is the minimal span among all $\operatorname{rssn}^{D}(c)$ of $G$. A graph admitting radio square sum D-distance coloring is called radio square sum D-distance graphs. We discussed a new concept radio square sum D-distance number of certain graphs.

KEYWORDS: Sum Labeling, Square Sum Labeling, Radio Square Sum D-Distance Number
\end{abstract}

Received: Feb 03, 2021; Accepted: Feb 23, 2021; Published: Mar 17, 2021; Paper Id.: IJMCARJUN20213

\section{INTRODUCTION}

Radio labelling is an extention of distance two labelling which is motivated by channel assignment problem introduced by Hale[5].By a graph $\mathrm{G}=(\mathrm{V}(\mathrm{G}), \mathrm{E}(\mathrm{G}))$. We mean a simple, connected graph without loops or multiple edges. The concept of square sum labeling was introduced by V. Ajitha [6].

We assign positive integers to the vertices of graph subject to a restriction concerning the distance between vertices $\mathrm{u}$ and $\mathrm{v}$. Let graph $\mathrm{G}$ with diameter and number to an extent that $1 \leq \mathrm{k} \leq \mathrm{d}$. The radio $\mathrm{k}$-coloring of $\mathrm{G}$ is a task to assign $\mathrm{c}$ of colors to vertices of $\mathrm{G}$ for following condition that $\mathrm{d}(\mathrm{u}, \mathrm{v})+|c(u)-c(v)| \geq 1+\mathrm{k}$ among all $\mathrm{u}, \mathrm{v}$ of G, Chatrand et al, [2,3] was presented radio labeling of graphs. D. Reddy babu presented the idea of D-distance et al. [11]

Radio D-distance and Radio mean D-distance labeling was presented by Dr.T,Nicholas and Dr .K.John Bosco in 2017 [7,8].. The connected $\mathrm{u}-\mathrm{v}$ path $\mathrm{s}$ is defined as $l^{D}(s)=l(s)+\operatorname{deg}(v)+\operatorname{deg}(u)+\sum \operatorname{deg}(w)$ and $l(s)$ is the length of the path. D-distance of graph $\mathrm{G}$ is defined as $d^{D}(u, v)=\min \left\{l^{D}(s)\right\}$ where the minimum is taken over all $\mathrm{u}-\mathrm{v}$ paths $\mathrm{s}$ in $\mathrm{G}$.

We introduced the concept of radio square difference $D$-distance labeling of a graph $\mathrm{G}[9,10]$. In this we introduce a new concept radio square sum D-distance labelling of graphs.

\section{MAIN RESULT}

\section{Theorem .1.1.}

For $n$, Radio square sum D-distance number of complete graphs, $K_{n}, \operatorname{rssn}^{D}\left(K_{n}\right)=\mathrm{n}$. 


\section{Proof:}

Let $\mathrm{V}\left(K_{n}\right)=\left\{v_{1}, v_{2}, v_{3}, \ldots, v_{n}\right\}$ be the vertex set .

Then $d^{D}\left(v_{i}, v_{j}\right)=2 n-1$

$\operatorname{diam}^{D}\left(K_{n}\right)=2 n-1$

The radio square sum $D$-distance condition,

$d^{D}(u, v)+\left|[c(u)]^{2}+[c(v)]^{2}\right| \geq \operatorname{diam}^{D}(G)+1$, for all $(u, v)$ where $u \neq v$

$d^{D}\left(v_{i}, v_{j}\right)+\left|\left[c\left(v_{i}\right)\right]^{2}+\left[c\left(v_{j}\right)\right]^{2}\right| \geq \operatorname{diam}^{D}\left(K_{n}\right)+1$,

which implies $\left|\left[c\left(v_{i}\right)\right]^{2}+\left[c\left(v_{j}\right)\right]^{2}\right| \geq 1$.

Therefore $\operatorname{rssn}^{D}\left(K_{n}\right)=\mathrm{n}$.

\section{Theorem .1.2.}

For $n \geq 2$, Radio square sum $D$-distance number of star graphs $K_{1, n}, \operatorname{rssn}^{D}\left(K_{1, n}\right)=n+1$.

\section{Proof:}

Let $\mathrm{V}\left(K_{1, n}\right)=\left\{v_{0}, v_{1}, v_{2}, v_{2}, \ldots, v_{n}\right\}$

Then $d^{D}\left(v_{i}, v_{i+1}\right)=n+4,1 \leq i \leq n$.

So $\operatorname{diam}^{D}\left(K_{1, n}\right)=n+4$.

The radio square sum D-distance condition becomes, $d^{D}(u, v)+\left|[c(u)]^{2}+[c(v)]^{2}\right| \geq \operatorname{diam}^{D}\left(K_{1, n}\right)+1$

For $\left(v_{0}, v_{i}\right), d^{D}(u, v)+\left|\left[c\left(v_{0}\right)\right]^{2}+\left[c\left(v_{i}\right)\right]^{2}\right| \geq n+5$

$n+2+\left|[1]^{2}+(i+1)^{2}\right| \geq n+5$,

For $\left(v_{i}, v_{i+1}\right), d^{D}\left(v_{i}, v_{i+1}\right)+\left|\left[c\left(v_{i}\right)\right]^{2}+\left[c\left(v_{i+1}\right)\right]^{2}\right| \geq n+5$

$$
n+4+\left|(i+1)^{2}+(i+2)^{2}\right| \geq n+5
$$

Define the function $f$ as $f\left(v_{0}\right)=1$ and

$$
f\left(v_{i}\right)=i+1,1 \leq i \leq n
$$

Therefore $\operatorname{rssn}^{D}\left(K_{1, n}\right)=n+1$, if $n \geq 2$.

\section{Theorem.1.3}

For $n \geq 2$. The radio square sum D-distance number of subdivision $\operatorname{star}$ graph $\mathrm{S}\left(K_{1, n}\right)$,

$\operatorname{rsdn} n^{D}\left(S\left(K_{1,2}\right)\right)=2 n+1$

Proof:

Let $\mathrm{V}\left(S\left(K_{1, n}\right)\right)=\left\{v_{0}, v_{1}, v_{3}, \ldots, v_{n}, u_{1}, u_{2}, u_{3}, \ldots, u_{n}\right\}$, and 
$E\left(S\left(K_{1, n}\right)\right)=\left\{v_{0} v_{i}, v_{i} u_{i}, 1 \leq i \leq n\right\}$ where $v_{0}$ is the apex vertex.

Then $d^{D}\left(v_{i}, u_{i}\right)=4,1 \leq i \leq n$,

$$
\begin{gathered}
d^{D}\left(v_{0}, u_{i}\right)=n+5,1 \leq i \leq n, \\
d^{D}\left(v_{i}, v_{j}\right)=n+6,1 \leq i, j \leq n, \\
d^{D}\left(u_{i}, u_{j}\right)=n+10 \\
\operatorname{diam}^{D}\left(S\left(K_{1, n}\right)\right)=\mathrm{n}+10 .
\end{gathered}
$$

Case 1. When $\mathrm{n}$ is odd

$f\left(v_{0}\right)=1, f\left(u_{i}\right)=i+2, f\left(v_{i}\right)=n+i+1$

We check radio square sum D-distance condition ,

$$
\begin{gathered}
\text { For }\left(u_{i}, u_{j}\right), \quad d^{D}\left(u_{i}, u_{j}\right)+\left|\left[c\left(u_{i}\right)\right]^{2}+\left[c\left(u_{j}\right)\right]^{2}\right| \geq n+11 \\
n+10+\left|(i+2)^{2}+(j+2)^{2}\right| \geq n+11, \\
\text { For }\left(v_{i}, v_{j}\right), \quad d^{D}\left(v_{i}, v_{j}\right)+\left|\left[c\left(v_{i}\right)\right]^{2}+\left[c\left(v_{j}\right)\right]^{2}\right| \geq n+11 \\
n+6+\left|(n+i+1)^{2}+(n+j+1)^{2}\right| \geq n+11,
\end{gathered}
$$

$\operatorname{For}\left(v_{i}, u_{i}\right), d^{D}\left(v_{i}, u_{i}\right)+\left|\left[c\left(v_{i}\right)\right]^{2}+\left[c\left(u_{i}\right)\right]^{2}\right| \geq n+11$

$$
4+\left|(n+i+1)^{2}+(i+2)^{2}\right| \geq n+11,
$$

$\operatorname{For}\left(v_{i}, u_{i}\right), d^{D}\left(v_{i}, u_{i}\right)+\left|\left[c\left(v_{0}\right)\right]^{2}+\left[c\left(u_{i}\right)\right]^{2}\right| \geq n+11$

$$
n+5+\left|(1)^{2}+(i+2)^{2}\right| \geq n+11,
$$

Case 2.

When $n$ is even

$$
\begin{aligned}
& c\left(v_{0}\right)=1, \\
& c\left(u_{i}\right)=i+2, \\
& c\left(v_{i}\right)=n+i+2
\end{aligned}
$$

The radio square sum D-distance condition ,

$$
\begin{gathered}
d^{D}\left(u_{i}, u_{j}\right)+\left|\left[c\left(u_{i}\right)\right]^{2}+\left[c\left(u_{j}\right)\right]^{2}\right| \geq n+11 \\
n+10+\mid\left[(i+2)^{2}+(j+2)^{2} \mid \geq n+11,\right. \\
d^{D}\left(v_{i}, v_{j}\right)+\left|\left[c\left(v_{i}\right)\right]^{2}+\left[c\left(v_{j}\right)\right]^{2}\right| \geq n+11 \\
n+6+\left|(n+i+2)^{2}+(n+j+2)^{2}\right| \geq n+11, \\
d^{D}\left(v_{i}, u_{i}\right)+\left|\left[c\left(v_{i}\right)\right]^{2}+\left[c\left(u_{i}\right)\right]^{2}\right| \geq n+11
\end{gathered}
$$




$$
4+\left|(n+i+2)^{2}+(i+2)^{2}\right| \geq n+11,
$$

$d^{D}\left(v_{0}, u_{i}\right)+\left|\left[c\left(v_{0}\right)\right]^{2}+\left[c\left(u_{i}\right)\right]^{2}\right| \geq n+11$,

$$
n+5+\left|(1)^{2}+(i+2)^{2}\right| \geq n+11,
$$

Then $c\left(v_{i}\right)=n+i+1,1 \leq i \leq n$ and

$$
c\left(u_{i}\right)=i+2,1 \leq i \leq n .
$$

Hence, $\operatorname{rssn}^{D}\left(S\left(K_{1, n}\right)\right)=\left\{\begin{array}{l}2 n+1, \text { if } n \text { is odd } \\ 2 n+2, \text { if } n \text { is even }\end{array}\right.$

\section{Theorem 1.4.}

Radio sum D-distance number of Fan graphs, $\operatorname{rssn}^{D}\left(F_{n}\right)=\left\{\begin{array}{cc}n+1, & \text { if } 2 \leq n \leq 10 \\ n+2, & \text { if } n \geq 11\end{array}\right.$

\section{Proof:}

Let $\mathrm{V}\left(F_{n}\right)=\left\{v_{0}, v_{1}, v_{2}, \ldots, v_{n}\right\}$

It is clear that $\operatorname{diam}^{D}\left(F_{n}\right)=n+8$,

We verify the radio square sum D-distance condition,

$d^{D}(u, v)+\left|[c(u)]^{2}+[c(v)]^{2}\right| \geq \operatorname{diam}^{D}\left(F_{n}\right)+1$

$v_{0}=1$,

$v_{2 i+1}=i+3,0 \leq i \leq \frac{n}{2}-1$,

$v_{2 i}=n-i+3,1 \leq i \leq \frac{n}{2}$,

If $v_{0}$ and $v_{2 i+1}$ are adjacent,

$d^{D}\left(v_{0}, v_{2 i+1}\right)+\left|\left[c\left(v_{0}\right)\right]^{2}+\left[c\left(v_{2 i+1}\right)\right]^{2}\right| \geq n+9$

$$
\mathrm{n}+4+\left|1^{2}+(i+3)^{2}\right| \geq n+9,
$$

If $v_{0}$ and $v_{2 i}$ are adjacent,

$$
\begin{aligned}
& d^{D}\left(v_{0}, v_{2 i}\right)+\left|\left[c\left(v_{0}\right)\right]^{2}+\left[c\left(v_{2 i}\right)\right]^{2}\right| \geq n+9 \\
& n+3+\left|(1)^{2}+(n-i+3)^{2}\right| \geq n+9,
\end{aligned}
$$

If $v_{2 i}$ and $v_{2 i+1}$ are adjacent

$$
\begin{aligned}
& d^{D}\left(v_{2 i+1}, v_{2 i}\right)+\left|\left[c\left(v_{2 i+1}\right)\right]^{2}+\left[c\left(v_{2 i}\right)\right]^{2}\right| \geq n+9 \geq(n-i+3)^{2} \mid \geq n+9 \\
& 6+\mid(i+3)^{2}+(n-9 \\
& d^{D}\left(v_{2 i+1}, v_{2 i}\right)+\left|\left[c\left(v_{2 i+1}\right)\right]^{2}+\left[c\left(v_{2 i}\right)\right]^{2}\right| \geq n+9 \\
& 5+\left|(i+3)^{2}+(n-i+3)^{2}\right| \geq n+9
\end{aligned}
$$

If $v_{i}$ and $v_{j}$ are non adjacent, 


$$
\begin{aligned}
d^{D}\left(v_{2 i+1}, v_{2 i}\right)+\mid\left[c\left(v_{2 i+1}\right)\right]^{2} & +\left[c\left(v_{2 i}\right)\right]^{2} \mid \geq n+9 \\
n & +8+\left|(i+3)^{2}+(n-i+3)^{2}\right| \geq n+9
\end{aligned}
$$

Hence, $r \operatorname{ssn}^{D}\left(F_{n}\right)=\left\{\begin{array}{cc}n+1, & \text { if } 2 \leq n \leq 10 \\ n+2, & \text { if } n \geq 11\end{array}\right.$

\section{Theorem:1.5.}

For $m \geq 5$, Radio square sum D-distance number of Book with triangle page graphs,

$$
K_{2}+m K_{1}, \operatorname{rssn}^{D}\left(K_{2}+m K_{1}\right)=m+n-3
$$

\section{Proof:}

Let $V\left(K_{2}+m K_{1}\right)=\left\{v_{1}, v_{2}, u_{1}, u_{2}, u_{3}, \ldots, u_{m}\right\}$ and

$$
E\left(K_{2}+m K_{1}\right)=\left\{v_{1} v_{2}, v_{1} u_{i}, v_{2} u_{i} 1 \leq i \leq n\right\},
$$

Then $d^{D}\left(v_{i}, u_{j}\right)=m+4,1 \leq i, j \leq m$,

$$
\begin{aligned}
& d^{D}\left(v_{1}, v_{2}\right)=2 m+3, \\
& d^{D}\left(u_{i}, u_{j}\right)=m+7,1 \leq i, j \leq n,
\end{aligned}
$$

and $\operatorname{diam}^{D}\left(K_{2}+m K_{1}\right)=2 \mathrm{~m}+3$.

We check radio square sum D-distance condition is

$$
\begin{gathered}
d^{D}\left(u_{i}, u_{j}\right)+\left|\left[c\left(u_{i}\right)\right]^{2}+\left[c\left(u_{j}\right)\right]^{2}\right| \geq 2 m+4 \\
n+10+\left|(m+i-3)^{2}+(m+j-3)^{2}\right| \geq 2 m+4, \\
d^{D}\left(v_{1}, v_{2}\right)+\left|c\left(v_{1}\right)^{2}+c\left(v_{2}\right)^{2}\right| \geq 2 m+4 \\
2 m+3+\left|(1)^{2}+(2)^{2}\right| \geq 2 m+4, \\
d^{D}\left(v_{i}, u_{j}\right)+\left|c\left(v_{i}\right)^{2}+c\left(u_{j}\right)^{2}\right| \geq 2 m+4 \\
m+7+\left|(i)^{2}+(m+j-3)^{2}\right| \geq 2 m+4,
\end{gathered}
$$

Hence, $r \operatorname{ssn}^{D}\left(K_{2}+m K_{1}\right)=m+n-3$ if $m \geq 5$.

\section{Theorem.1.6.}

For $\mathrm{n} \geq 3$, Radio square sum D-distance number of comb graphs, $r \operatorname{ssn}^{D}\left(P_{n} \odot K_{1}\right)=2 \mathrm{n}$

Proof:

Let $\mathrm{V}\left(P_{n} \odot K_{1}\right)=\left\{v_{i}, u_{i}, 1 \leq i \leq n\right\}$ and

$\mathrm{E}=\left\{v_{i}, v_{i+1}, v_{i}, u_{i}, 1 \leq i \leq n\right\}$

Let $c\left(u_{1}\right)=1$,

$c\left(u_{n}\right)=2$ and 


$$
\begin{aligned}
& c\left(u_{i}\right)=i+1, \\
& c\left(v_{i}\right)=2 n-i+1
\end{aligned}
$$

We verify the radio square sum D-distance condition,

$$
\begin{aligned}
& d^{D}(u, v)+\left|[c(u)]^{2}+[c(v)]^{2}\right| \geq \operatorname{diam}^{D}\left(F_{n}\right)+1 \\
& d^{D}\left(u_{i}, u_{j}\right)+\left|\left[c\left(u_{i}\right)\right]^{2}+\left[c\left(u_{j}\right)\right]^{2}\right| \geq 4 n+2, \\
& 4 n+1+\left|(i+1)^{2}+(j+1)^{2}\right| \geq 4 n+2, \\
& d^{D}\left(u_{i}, u_{j}\right)+\left|\left[c\left(u_{i}\right)\right]^{2}+\left[c\left(u_{j}\right)\right]^{2}\right| \geq 4 n+2 \\
& 4 n-6+\left|(i+1)^{2}+(j+1)^{2}\right| \geq 4 n+2,
\end{aligned}
$$$$
d^{D}\left(u_{1}, u_{2}\right)+\left|\left[c\left(u_{1}\right)\right]^{2}+\left[c\left(u_{2}\right)\right]^{2}\right| \geq 4 n+2
$$

$$
4 n+1+\left|(1)^{2}+(2)^{2}\right| \geq 4 n+2,
$$$$
d^{D}\left(v_{i}, v_{j}\right)+\left|\left[c\left(v_{i}\right)\right]^{2}+\left[c\left(v_{j}\right)\right]^{2}\right| \geq 4 n+2
$$$$
n+6+\left|(2 n-i+1)^{2}+(2 n-j+1)^{2}\right| \geq 4 n+2,
$$$$
d^{D}\left(v_{i}, v_{j}\right)+\left|\left[c\left(v_{i}\right)\right]^{2}+\left[c\left(v_{j}\right)\right]^{2}\right| \geq 4 n+2
$$$$
4 n-3+\left|(2 n-i+1)^{2}+(2 n-j+1)^{2}\right| \geq 4 n+2,
$$$$
d^{D}\left(v_{i}, v_{j}\right)+\left|\left[c\left(v_{i}\right)\right]^{2}+\left[c\left(v_{j}\right)\right]^{2}\right| \geq 4 n+2
$$

$$
4 n-7+\left|(2 n-i+1)^{2}+(2 n-j+1)^{2}\right| \geq 4 n+2,
$$

$d^{D}\left(u_{i}, u_{j}\right)+\left|c\left(u_{i}\right)^{2}+c\left(u_{j}\right)^{2}\right| \geq 4 n+2$

$$
4 n-7+\left|(i+1)^{2}+(j+1)^{2}\right| \geq 4 n+2,
$$

Hence, $\operatorname{rssn}^{D}\left(P_{n} \odot K_{1}\right)=2 \mathrm{n}$ if $n \geq 3$

\section{REFERENCES}

1. F. Buckley and F. Harary, Distance in Graphs,Addition- Wesley, Redwood City, CA, 1990.

2. G.Chartrand, D. Erwinn, F. Harary, and P. Zhang, "Radio labeling of graphs," Bulletin of the Institute of Combinatorics and Its Applications, vol. 33, pp. 77-85, 2001.

3. G.Chartrand, D. Erwin, and P. Zhang, Graph labeling problem suggested by FM channel restrict ions, Bull. Inst. Combin. Appl., 43, 43-57(2005).

4. J.A. Gallian, A dynamic survey of graph labeling, Electron. J. Combin. 19 (2012) \#Ds6.

5. W.K. Hale, Frequency assignment: Theory and applications, Proc. IEEE 68 (1980), pp.1497-1514.

6. V.Ajitha,s.Arumugam,K.AGermina, On square sum graphs AKCE, International Journal of Graphs and CombinatoricsAKCE6 No.1(2009) pp.(1-10) 
7. T.Nicholas and K.JohnBosco, Radio D-distance number of some graphs, International Journal of Engineering \& Scientific Research Vol.5 Issue 2, February 2017, ISSN: 2347-6532.

8. T.Nicholas, K.JohnBosco and M. Antony, Radio mean D-distance labeling of some graphs, International Journal of Engineering \& Scientific Research Vol.5 Issue 2, February 2017, ISSN: 2347-6532. publication in ArsCombinatoria.

9. K.Johnbosco, G.Vishma George, On Radio square Difference D-distance number of some standard graphs, IJRAR January 2021, Volume 8, Issue 1 www.ijrar.org (E-ISSN 2348-1269, P-ISSN 2349-5138)

10. K.Johnbosco, G.Vishma George, On Radio square difference Dd-distance number of cycle related Graphs, JETIR January 2021, Volume 8, Issue 1 www.jetir.org (ISSN-2349-5162)

11. Reddy Babu, D., Varma, P.L.N., D-distance in graphs, Golden Research Thoughts, 2(2013), 53-58. 
